\title{
Editorial \\ Roles for estrogen and progesterone in breast cancer prevention
}

\author{
D Joseph Jerry 1,2
}

\begin{abstract}
1Department of Veterinary \& Animal Sciences, University of Massachusetts-Amherst, 161 Holdsworth Way, Amherst, Massachusetts 01003, USA 2Pioneer Valley Life Sciences Institute, 3601 Main Street, Springfield, Massachusetts 01199, USA
\end{abstract}

Correspondence: D Joseph Jerry, jjerry@vasci.umass.edu

Published: 21 March 2007

This article is online at http://breast-cancer-research.com/content/9/2/102

(c) 2007 BioMed Central Ltd

Breast Cancer Research 2007, 9:102 (doi:10.1186/bcr1659)

\begin{abstract}
Prevention has long been the holy grail of breast cancer research. The significant reduction in breast cancer risk afforded by a fullterm pregnancy early in life suggests the great potential of preventive strategies. In contrast to the risks associated with prolonged exposures, exogenous estrogen and progesterone for short durations can mimic the protective effects of pregnancy in carcinogen-induced mammary tumor models. Rajkumar and coworkers have now demonstrated that these hormones protect mice from mammary tumors initiated by a spectrum of oncogenic alterations that are common in breast cancers. Although differences between rodent models and humans remain, the results reveal that exogenous estrogen and progesterone potently inhibit tumorigenesis through multiple pathways and establish a foundation for strategies to prevent breast cancer.
\end{abstract}

\section{Introduction}

Estrogen and progesterone play leading roles in orchestrating proper development and function of breast tissue. High levels of these hormones are associated with pregnancy and appear to be responsible for the diminished risk for breast cancer among women following a full-term pregnancy. Exogenous estrogen and progesterone are sufficient to mimic the protective effects of parity and suppress mammary tumors in rodents exposed to the carcinogen dimethylbenz $(\alpha)$ anthracene (DMBA). However, the relevance of these results has been debated because the relationship between polyaromatic hydrocarbons and the oncogenic events observed in breast cancers remain unclear.

To address this, investigators from the laboratories of Rajkumar and Medina [1] have examined the effects of exogenous estrogen and progesterone in mouse models that bear lesions in clinically relevant pathways. Because overexpression of the HER2/neu oncogene is observed in up to $30 \%$ of breast cancers, responses to treatment with estrogen and progesterone were analyzed in mice overexpressing HER2/neu (MMTV-neu mice). Exogenous estrogen and progesterone reduced the incidence of mammary tumors in these mice by more than 60\%. The p53 tumor suppressor pathway is also commonly disrupted in breast cancers. Mice bearing mammary epithelial transplants that have homozygous deletions in the mouse p53 gene $\left(\operatorname{Trp} 53^{-/}\right)$provide a model for loss of p53. When exogenous hormones were administered to mice just after puberty (age 5 to 7 weeks), the incidence of tumors in Trp53\% mammary tissues was reduced by at least $70 \%$. The protective effect was preserved even when estrogen and progesterone were administered to older mice (age 23 to 25 weeks), indicating that the timing of treatments was not critical. The exogenous hormones did not affect development of preneoplastic lesions but rather prevented their apparent progression to tumors. Together, the experiments demonstrate that a 14-day treatment with exogenous estrogen and progesterone can render the mammary epithelium resistant to diverse oncogenic pathways.

\section{Mechanisms implicated}

Both p53-dependent and p53-independent pathways appear to contribute to hormone-mediated protection in mammary tissue. Acute cellular responses to genotoxic agents include cell cycle arrest and apoptosis mediated by the p53 tumor suppressor protein. In addition to the acute genotoxic effects, carcinogens commonly cause activating mutations in ras oncogenes in mammary tissue $[2,3]$, which can initiate senescence through p53-dependent pathways [4]. Because p53-mediated responses to DNA damage are increased dramatically following treatment with estrogen and progesterone [5] and parity failed to prevent tumors in $\operatorname{Trp} 53^{\%}$ mammary transplants of mice exposed to DMBA, the p53 pathway appeared to be essential [6]. However, Rajkumar and coworkers [1] have now demonstrated that p53-independent pathways are also activated by exogenous estrogen and progesterone, resulting in a greater than $70 \%$ reduction in spontaneous mammary tumors in p53-deficient mammary tissues.

The distinction between DMBA-induced tumors and spontaneous tumors appears critical and suggests that there 
are differences in the carcinogenic mechanisms. The p53 pathway is probably crucial for antagonizing oncogenes that are activated in DMBA-induced tumors (for example, ras). In contrast, tumors in $\operatorname{Trp} 53^{\%}$ mammary epithelium may depend on errors in chromosome segregation, which are common in $\mathrm{Trp} 3^{\%}$ tissues and would promote loss of additional tumor suppressors. In this setting, the reduced proliferation following treatment with exogenous estrogen and progesterone [1] may reduce the opportunity for deletion of tumor suppressor pathways by this mechanism.

Hormonal stimulation causes numerous local and systemic alterations, which could render the mammary epithelium resistant to tumorigenesis and not require p53. Substantial changes in the extracellular matrix and reorganization of the stroma $[7,8]$ occur in parous breast tissue that have the potential to restrain tumorigenesis $[9,10]$. Alterations in circulating levels of endocrine hormones and growth factors following pregnancy have also been implicated. In addition, sex hormones have potent effects on immune function [11] that can affect tumor progression. Although p53-independent pathways remain to be identified, they represent important targets for prevention because they would be effective even in preinvasive lesions in which p53 had been lost.

\section{A bumpy road to the clinic}

Although rodent models mimic the protective effects of parity in women, significant differences exist. The levels of estrogen and progesterone during the last trimester of pregnancy are much greater in women, often exceeding $10,000 \mathrm{pg} / \mathrm{ml}$, as compared with about $70 \mathrm{pg} / \mathrm{ml}$ in mice. Thus, it is possible that much higher levels of estrogen will be required for successful prophylaxis in women. If cells bearing oncogenic lesions accumulate over time, then the high levels of estrogen required for prophylaxis in women may promote progression of the initiated cells. This may explain why the protective effect of parity is greatest among younger women [12]. Because elevated levels of estrogen and progesterone have been associated with increased risk for breast cancer [13], their promotional effects may exceed the inhibitory effects when they are administered beyond the teenage years. This concern would be mitigated if short-term treatment with exogenous estrogen and progesterone proves to be sufficient for prophylaxis. The stroma within which the mammary epithelium is embedded also differs substantially between mouse and human, and so may utilize very different signaling pathways. Given the need to establish minimum doses of hormones and duration of exposure sufficient for prophylaxis and the prospect that interventions may target women aged under 25 years, treatment strategies will face rigorous scrutiny, even if they are limited to high-risk populations.

\section{Conclusion}

In spite of significant differences between rodent models and human breast tissue, the fact remains that parity is protective in both. A growing body of evidence suggests that parity reduces risk, even among women bearing heritable mutations in BRCA1 and BRCA2 [14]. Thus, it appears that a full-term pregnancy engages multiple pathways that effectively antagonize tumors resulting from a spectrum of underlying oncogenic lesions. The signals initiated by pregnancy appear to converge upon a set of pathways that are shared by both rodent mammary and human breast tissues. Therapeutic agents targeting these critical effector pathways would make it possible to exploit the protective effects while avoiding the potential risks posed by exogenous hormones. Transcriptional profiling of mammary tissues from nulliparous and parous rodents has revealed a set of differentially expressed candidate genes [15]. A much larger list of effectors is likely to emerge as genome-wide transcriptional profiles become available for model organisms and human breast tissues. The datasets are likely to yield a rich harvest of pathways that contribute to the overall protection afforded by parity. The work by Rajkumar and coworkers [1] provides confidence that the underlying pathways will have the ability to overcome a variety of oncogenic alterations in epithelial cells and will invigorate the search for effective strategies to prevent breast cancer.

\section{Competing interests}

The author declares that they have no competing interests.

\section{References}

1. Rajkumar L, Kittrell FS, Guzman RC, Brown PH, Nandi S, Medina $\mathrm{D}$ : Hormone-induced protection of mammary tumorigenesis in genetically engineered mouse models. Breast Cancer Res 2007, 9:R12.

2. Kumar R, Medina D, Sukumar S: Activation of H-ras oncogenes in preneoplastic mouse mammary tissues. Oncogene 1990, 5: 1271-1277.

3. Zarbl H, Sukumar S, Arthur AV, Martin-Zanca D, Barbacid M: Direct mutagenesis of Ha-ras-1 oncogenes by $\mathrm{N}$-nitroso- $\mathrm{N}$ methylurea during initiation of mammary carcinogenesis in rats. Nature 1985, 315:382-385.

4. Yaswen P, Campisi J: Oncogene-induced senescence pathways weave an intricate tapestry. Cell 2007, 128:233-234

5. Becker KA, Lu S, Dickinson ES, Dunphy KA, Mathews L, Schneider SS, Jerry DJ: Estrogen and progesterone regulate radiation-induced p53 activity in mammary epithelium through TGF-beta-dependent pathways. Oncogene 2005, 24:63456353.

6. Medina D, Kittrell FS: p53 function is required for hormonemediated protection of mouse mammary tumorigenesis. Cancer Res 2003, 63:6140-6143.

7. Haslam SZ, Woodward TL: Host microenvironment in breast cancer development: epithelial-cell-stromal-cell interactions and steroid hormone action in normal and cancerous mammary gland. Breast Cancer Res 2003, 5:208-215.

8. Schedin P, Mitrenga T, McDaniel S, Kaeck M: Mammary ECM composition and function are altered by reproductive state. Mol Carcinog 2004, 41:207-220.

9. Proia DA, Kuperwasser C: Stroma: tumor agonist or antagonist. Cell Cycle 2005, 4:1022-1025.

10. Wang F, Hansen RK, Radisky D, Yoneda T, Barcellos-Hoff MH, Petersen OW, Turley EA, Bissell MJ: Phenotypic reversion or death of cancer cells by altering signaling pathways in threedimensional contexts. J Natl Cancer Inst 2002, 94:1494-1503.

11. Verthelyi D: Sex hormones as immunomodulators in health and disease. Int Immunopharmaco/ 2001, 1:983-993.

12. Rosner B, Colditz GA, Willett WC: Reproductive risk factors in a prospective study of breast cancer: the Nurses' Health Study. Am J Epidemiol 1994, 139:819-835.

13. Eliassen AH, Missmer SA, Tworoger SS, Spiegelman D, Barbieri $\mathrm{RL}$, Dowsett M, Hankinson SE: Endogenous steroid hormone 
concentrations and risk of breast cancer among premenopausal women. J Nat/ Cancer Inst 2006, 98:1406-1415.

14. Antoniou AC, Shenton A, Maher ER, Watson E, Woodward E, Lalloo F, Easton DF, Evans DG: Parity and breast cancer risk among BRCA1 and BRCA2 mutation carriers. Breast Cancer Res 2006, 8:R72.

15. Blakely CM, Stoddard AJ, Belka GK, Dugan KD, Notarfrancesco KL, Moody SE, D'Cruz CM, Chodosh LA: Hormone-induced protection against mammary tumorigenesis is conserved in multiple rat strains and identifies a core gene expression signature induced by pregnancy. Cancer Res 2006, 66:6421. 6431. 